\title{
Existence and Uniqueness of Solutions for the Cauchy-Type Problems of Fractional Differential Equations
}

\author{
Chunhai Kou, ${ }^{1}$ Jian Liu, ${ }^{1}$ and Yan Ye $^{2}$ \\ ${ }^{1}$ Department of Applied Mathematics, Donghua University, Shanghai 201620, China \\ ${ }^{2}$ College of Information Sciences and Technology, Donghua University, Shanghai 201620, China
}

Correspondence should be addressed to Chunhai Kou, kouchunhai@hotmail.com

Received 30 October 2009; Revised 21 January 2010; Accepted 25 January 2010

Academic Editor: Guang Zhang

Copyright (C) 2010 Chunhai Kou et al. This is an open access article distributed under the Creative Commons Attribution License, which permits unrestricted use, distribution, and reproduction in any medium, provided the original work is properly cited.

By using the Banach fixed point theorem and step method, we study the existence and uniqueness of solutions for the Cauchy-type problems of fractional differential equations. Meanwhile, by citing some counterexamples, it is pointed out that there exist a few defects in the proofs of the known results.

\section{Introduction}

Recently, fractional differential equations are applied widely in various fields of science and engineering. Regarding applications of fractional differential equations, we refer to [115] and references cited therein. However, the investigation of basic theory of fractional differential equations is still not complete, and there is a great deal of work which needs to be done. Most of the investigations in this field involve the existence and uniqueness of solutions to fractional differential equations on the finite interval $[a, b]$. In 1938, Pitcher and Sewell [16] first considered the nonlinear fractional differential equation

$$
\left(D_{a+}^{\alpha} y\right)(x)=f[x, y(x)]
$$

with the following initial conditions:

$$
\left(D_{a+}^{\alpha-k} y\right)(a+)=b_{k}, \quad b_{k} \in \mathbb{R},(k=1, \ldots, n, n=-[-\alpha]),
$$


where $0<\alpha<1$, and $D_{a+}^{\alpha}$ is Riemann-Liouville fractional derivative. Barrett [17], in 1954, first considered the Cauchy-type problem for the linear fractional differential equation

$$
\left(D_{a+}^{\alpha} y\right)(x)-\lambda y(x)=f(x), \quad(n-1 \leq R(\alpha)<n, \alpha \neq n-1),
$$

with the same initial conditions (1.2). Afterwards, there is a great deal of work about the basic theory [18-27]. In [28], Kilbas et al. summarized systematically the main results.

In this paper we consider the cauchy problem (1.1)-(1.2); here $D_{a+}^{\alpha}$ can be RiemannLiouville fractional derivative, and Hadamard-type fractional derivative. We establish some results about the existence and uniqueness of solution of (1.1)-(1.2). By the way, we will point out that there exist several defects in the proofs of the related theorems of [28].

This paper is organized as follows: in Section 2, we introduce some preliminaries and notations; main results are proved in Section 3; in Section 4, by citing several counterexamples, we will point out the defects in [28]; Section 5 is a brief summary of this paper.

\section{Preliminaries and Notations}

In this section, we introduce some basic definitions and notations about fractional calculus. Meanwhile, several known theorems are given, which are useful in this paper.

Definition 2.1 (see [28]). Let $\Omega=(a, b](-\infty<a<b<\infty)$ be a finite interval on the real axis $\mathbb{R}$. The Riemann-Liouville left-sided fractional integral $I_{a+}^{\alpha} g$ of the function $g$ with order $\alpha \in \mathbb{R}(\alpha>0)$ is defined by

$$
\left(I_{a+g}^{\alpha} g\right)(x)=\frac{1}{\Gamma(\alpha)} \int_{a}^{x} \frac{g(t) d t}{(x-t)^{1-\alpha}}, \quad(x>a),
$$

where the real function $g$ is defined on the interval $\Omega$ and the right-side integral of the above equality is assumed to make sense.

Definition 2.2 (see [28]). Let $\Omega=(a, b](-\infty<a<b<\infty)$ be a finite interval on the real axis $\mathbb{R}$. The Riemann-Liouville left-sided fractional derivative $D_{a+}^{\alpha} g$ of the function $g$ with order $\alpha \in \mathbb{R}(\alpha \geq 0)$ is defined by

$$
\begin{aligned}
\left(D_{a+}^{\alpha} g\right)(x) & =\left(\frac{d}{d x}\right)^{n}\left(I_{a+}^{n-\alpha} g\right)(x) \\
& =\frac{1}{\Gamma(n-\alpha)}\left(\frac{d}{d x}\right)^{n} \int_{a}^{x} \frac{g(t) d t}{(x-t)^{\alpha-n+1}}, \quad(x>a ; n=-[-\alpha]),
\end{aligned}
$$

where the real function $g$ is defined on the interval $\Omega$ and the right side of the above equality is assumed to make sense. 
Definition 2.3. Assume that $f[x, y]$ is defined on the set $(a, b] \times G(G \subset \mathbb{R}) . f[x, y]$ is said to satisfy Lipschitzian condition with respect to the second variable, if for all $x \in(a, b]$ and for any $y_{1}, y_{2} \in G$ one has

$$
\left|f\left[x, y_{1}\right]-f\left[x, y_{2}\right]\right| \leq A\left|y_{1}-y_{2}\right|
$$

where $A>0$ does not depend on $x \in(a, b]$.

Definition 2.4 (see [28]). Let $n-1<\alpha \leq n(n \in N)$, then the space $C_{n-\alpha}^{\alpha}[a, b]$ is defined by

$$
C_{n-\alpha}^{\alpha}[a, b]=\left\{y(x) \in C_{n-\alpha}[a, b]:\left(D_{a+}^{\alpha} y\right)(x) \in C_{n-\alpha}[a, b]\right\}
$$

Here $C_{n-\alpha}[a, b]$ is a weighted space of continuous functions

$$
C_{n-\alpha}[a, b]=\left\{g:(a, b] \longrightarrow R:(x-a)^{n-\alpha} g(x) \in C[a, b]\right\}
$$

and $D_{a+}^{\alpha}$ is the Riemann-Liouville fractional derivative.

In the space $C_{n-\alpha}[a, b]$, we define the norm $\|g\|_{C_{n-\alpha}}=\left\|(x-a)^{n-\alpha} g(x)\right\|_{C}$.

Definition 2.5 (see [28]). Let $(a, b)(0<a<b \leq \infty)$ be a finite or infinite interval of the halfaxis $\mathbb{R}^{+}$. The Hadamard type left-sided fractional integral $\partial_{a+}^{\alpha} h$ of the function $h$ with order $\alpha \in \mathbb{R}(\alpha>0)$ is defined by

$$
\left(\partial_{a+}^{\alpha} h\right)(x)=\frac{1}{\Gamma(\alpha)} \int_{a}^{x}\left(\ln \frac{x}{t}\right)^{\alpha-1} \frac{h(t) d t}{t}, \quad(a<x<b),
$$

where $h:(a, b) \rightarrow \mathbb{R}$ and the right-side integral of the above equality is assumed to make sense.

Definition 2.6 (see [28]). Let $\delta=x D(D=d / d x)$ be the $\delta$-derivative. The Hadamard leftsided fractional derivative $\Phi_{a+}^{\alpha} y$ of the function $y$ on $(a, b)$ with order $\alpha \in \mathbb{R}(\alpha \geq 0)$ is defined by

$$
\begin{aligned}
\left(\Phi_{a+}^{\alpha} y\right)(x) & =\delta^{n}\left(\partial_{a+}^{n-\alpha} y\right)(x) \\
& =\frac{1}{\Gamma(n-\alpha)}\left(x \frac{d}{d x}\right)^{n} \int_{a}^{x}\left(\ln \frac{x}{t}\right)^{n-\alpha-1} \frac{y(t) d t}{t}, \quad(a<x<b ; n=-[-\alpha]),
\end{aligned}
$$

where $y:(a, b) \rightarrow \mathbb{R}, \delta^{n}=\underbrace{\delta \cdots \delta}_{n}$, and the right side of the above equality is assumed to make sense.

Definition 2.7 (see [28]). Let $n-1<\alpha \leq n(n \in N), 0<a<b<+\infty$, and $0 \leq \gamma<1$. The space $C_{\delta ; n-\alpha, \gamma}^{\alpha}[a, b]$ is defined by

$$
C_{\delta ; n-\alpha, \gamma}^{\alpha}[a, b]=\left\{y(x) \in C_{n-\alpha, \ln }[a, b]:\left(\Phi_{a+}^{\alpha} y\right) \in C_{\gamma, \ln }[a, b]\right\}
$$


where $\Phi_{a+}^{\alpha}$ is a Hadamard left-sided fractional derivative, and $C_{\gamma, \ln }[a, b]$ is a weighted space of continuous functions

$$
C_{\gamma, \ln }[a, b]=\left\{g:(a, b] \longrightarrow \mathbb{R}:\left(\ln \frac{x}{a}\right)^{\gamma} g(x) \in C[a, b]\right\}
$$

In the space $C_{\gamma, \ln }[a, b]$, we define the norm $\|g\|_{C_{\gamma, \mathrm{ln}}}=\left\|(\ln (x / a))^{\gamma} g(x)\right\|_{C}$.

Theorem 2.8 (see [28]). Let $\alpha>0, n=-[-\alpha]$. Let $f:(a, b] \times \mathbb{R} \rightarrow \mathbb{R}$ be a function such that $f[x, y(x)] \in C_{n-\alpha}[a, b]$ for any $y(x) \in C_{n-\alpha}[a, b]$. If $y(x) \in C_{n-\alpha}[a, b]$, then $y(x)$ satisfies the relations:

$$
\begin{aligned}
\left(D_{a+}^{\alpha} y\right)(x) & =f[x, y(x)], \quad(\alpha>0), \\
\left(D_{a+}^{\alpha-k} y\right)(a+) & =b_{k}, \quad b_{k} \in \mathbb{R}, \quad(k=1, \ldots, n=-[-\alpha]),
\end{aligned}
$$

if and only if $y(x)$ satisfies the Volterra integral equation

$$
y(x)=y_{0}(x)+\frac{1}{\Gamma(\alpha)} \int_{a}^{x} \frac{f[t, y(t)] d t}{(x-t)^{1-\alpha}}, \quad(x>a)
$$

where

$$
y_{0}(x)=\sum_{j=1}^{n} \frac{b_{j}}{\Gamma(\alpha-j+1)}(x-a)^{\alpha-j}
$$

where $D_{a+}^{\alpha}$ is a Riemann-Liouville left-sided fractional derivative.

Theorem 2.9 (see [28]). (Banach Fixed Point Theorem) Let $(U, d)$ be a nonempty complete metric space, let $0 \leq \omega<1$, and let $T: U \rightarrow U$ be a map such that, for every $u, v \in U$, the relation

$$
d(T u, T v) \leq \omega d(u, v), \quad(0 \leq \omega<1)
$$

holds. Then the operator $T$ has a unique fixed point $u^{*} \in U$.

Theorem 2.10 (see [28]). Let $0<a<b<\infty, \alpha>0, n=-[-\alpha]$, and $0 \leq \gamma<1$. Let $f:(a, b] \times \mathbb{R} \rightarrow$ $\mathbb{R}$ be a function such that $f[x, y(x)] \in C_{\gamma, \ln }[a, b]$ for any $y(x) \in C_{\gamma, \ln }[a, b]$. If $y(x) \in C_{n-\alpha, \ln }[a, b]$, then $y(x)$ satisfies

$$
\begin{aligned}
\left(\Phi_{a+}^{\alpha} y\right)(x) & =f[x, y(x)], \quad(x>a), \\
\left(\Phi_{a+}^{\alpha-k} y\right)(a+) & =b_{k}, \quad b_{k} \in \mathbb{R}, \quad(k=1, \ldots, n ; n=-[-\alpha]),
\end{aligned}
$$


if and only if $y(x)$ satisfies the Volterra integral equation

$$
y(x)=\sum_{j=1}^{n} \frac{b_{j}}{\Gamma(n-j+1)}\left(\ln \frac{x}{a}\right)^{\alpha-j}+\frac{1}{\Gamma(\alpha)} \int_{a}^{x}\left(\ln \frac{x}{t}\right)^{\alpha-1} f[t, y(t)] \frac{d t}{t}, \quad(x>a),
$$

where $\boldsymbol{\Phi}_{a+}^{\alpha}$ is a Hadamard-type left-sided fractional derivative.

Remark 2.11. It should be worthy noting that the conditions in Theorems 2.8 and 2.10 are a little different from the ones in [28, pages 163, 213]. In [28], $G$ is an open set in $R$ and $f$ is assumed to be a function such that $f[x, y] \in C_{n-\alpha}[a, b]\left(C_{\gamma, \ln }[a, b]\right)$ for any $y \in G$. In fact, we think that such assumption is not complete for the proof of the related conclusion.

\section{Main Results}

In this section, we will establish several useful lemmas. It should be pointed out that, in [28], some analogous lemmas play important roles in the proofs of the related results. However, we have found out that there exist a few defects in these lemmas of [28], which means that the proofs of the related results in [28] are not complete. Several counterexamples will be given in Section 4. In a sense, our lemmas are to mend these cracks. Furthermore, several theorems about the existence and uniqueness of solution for the cauchy-type problem (2.10)-(2.11) will be given; then, in the sense of Hadamard fractional derivative, we have the similar result.

Lemma 3.1. Let $\gamma \in[0, \infty), a<c<b, g \in C_{\gamma}[a, c]$, and $g \in C[c, b]$. Then $g \in C_{\gamma}[a, b]$ and

$$
\|g\|_{C_{\gamma}[a, b]} \leq \max \left\{\|g\|_{C_{\gamma}[a, c]^{\prime}}(b-a)^{\gamma}\|g\|_{C[c, b]}\right\}
$$

Proof. Since $g \in C_{\gamma}[a, c]$ and $g \in C[c, b]$, then $g \in C(a, b]$ and $g \in C_{\gamma}[a, b]$. Now we prove the estimate. Because $g \in C_{\gamma}[a, b]$, there exists $x_{0} \in[a, b]$ such that

$$
\|g\|_{C_{\gamma}[a, b]}=\left|\left(x_{0}-a\right)^{\gamma} g\left(x_{0}\right)\right| .
$$

If $x_{0} \in[a, c]$, then

$$
\|g\|_{C_{Y}[a, b]} \leq\|g\|_{C_{Y}[a, c]} .
$$

If $x_{0} \in[c, b]$, then

$$
\|g\|_{C_{\gamma}[a, b]} \leq(b-a)^{\gamma}\|g\|_{C[c, b]} .
$$

Hence we have

$$
\|g\|_{C_{\gamma}[a, b]} \leq \max \left\{\|g\|_{C_{\gamma}[a, c]^{\prime}}(b-a)^{\gamma}\|g\|_{C[c, b]}\right\} .
$$

This completes the proof of Lemma 3.1. 
Lemma 3.2 (see [28]). If $\gamma \in \mathbb{R}(0 \leq \gamma<1)$, then the fractional integration operator $I_{a+}^{\alpha}$ with order $\alpha \in \mathbb{R}(\alpha>0)$ is a mapping from $C_{\gamma}[a, b]$ to $C_{\gamma}[a, b]$, and

$$
\left\|I_{a+}^{\alpha} g\right\|_{C_{r}} \leq(b-a)^{\alpha} \frac{\Gamma(1-\gamma)}{\Gamma(1+\alpha-\gamma)}\|g\|_{C_{\gamma^{\prime}}}
$$

here $I_{a+}^{\alpha}$ is a Riemann-Liouville fractional integral operator and $g \in C_{\gamma}[a, b]$.

Furthermore, we have the following conclusion.

Lemma 3.3. The fractional integration operator $I_{a+}^{\alpha}$ with order $\alpha \in \mathbb{R}(\alpha>0)$ is a mapping from $C[a, b]$ to $C[a, b]$, and

$$
\left\|I_{a+g}^{\alpha} g\right\|_{C} \leq \frac{(b-a)^{\alpha}}{\alpha \Gamma(\alpha)}\|g\|_{C^{\prime}}
$$

where $I_{a+}^{\alpha}$ is a Riemann-Liouville fractional integral operator and $g \in C[a, b]$.

Proof. Firstly we prove that if $g \in C[a, b]$, then $\left(I_{a+}^{\alpha} g\right)(x) \in C[a, b]$. For any $x \in[a, b]$ and $\Delta x>0, x+\Delta x \leq b$, we have

$$
\begin{aligned}
&\left|\left(I_{a+}^{\alpha} g\right)(x+\Delta x)-\left(I_{a+}^{\alpha} g\right)(x)\right|=\left|\frac{1}{\Gamma(\alpha)} \int_{a}^{x+\Delta x} \frac{g(t) d t}{(x+\Delta x-t)^{1-\alpha}}-\frac{1}{\Gamma(\alpha)} \int_{a}^{x} \frac{g(t) d t}{(x-t)^{1-\alpha}}\right| \\
& \leq \frac{1}{\Gamma(\alpha)}\left\{\left|\int_{a}^{x} g(t)\left[\frac{1}{(x+\Delta x-t)^{1-\alpha}}-\frac{1}{(x-t)^{1-\alpha}}\right] d t\right|\right. \\
&\left.\quad+\left|\int_{x}^{x+\Delta x} \frac{g(t)}{(x+\Delta x-t)^{1-\alpha}} d t\right|\right\} \\
& \leq \frac{\|g\|_{C[a, b]}}{\alpha \Gamma(\alpha)}\left\{\left[(x+\Delta x-a)^{\alpha}-(x-a)^{\alpha}\right]+(\Delta x)^{\alpha}+(\Delta x)^{\alpha}\right\} .
\end{aligned}
$$

It is easy to see that as $\Delta x \rightarrow 0^{+}$, we have

$$
\left|\left(I_{a+}^{\alpha} g\right)(x+\Delta x)-\left(I_{a+}^{\alpha} g\right)(x)\right| \longrightarrow 0 .
$$

Similarly, we can prove that as $\Delta x \rightarrow 0^{-}$, we have

$$
\left|\left(I_{a+}^{\alpha} g\right)(x+\Delta x)-\left(I_{a+}^{\alpha} g\right)(x)\right| \longrightarrow 0 .
$$

Thus $I_{a+}^{\alpha} g \in C[a, b]$. 
Now we prove the estimate. In fact

$$
\begin{aligned}
\left\|I_{a+g}^{\alpha}\right\|_{C[a, b]} & =\max _{x \in[a, b]}\left|\frac{1}{\Gamma(\alpha)} \int_{a}^{x} \frac{g(t) d t}{(x-t)^{1-\alpha}}\right| \\
& \leq \frac{\|g\|_{C[a, b]}}{\Gamma(\alpha)} \int_{a}^{x}(x-t)^{\alpha-1} d t \\
& \leq \frac{(b-a)^{\alpha}}{\alpha \Gamma(\alpha)}\|g\|_{C} .
\end{aligned}
$$

This completes the proof of Lemma 3.3.

Lemma 3.4. Let $\gamma \in[0, \infty), 0<a<c<b<\infty, g \in C_{\gamma, \ln }[a, c]$ and $g \in C[c, b]$. Then $g \in$ $C_{\gamma, \ln }[a, b]$ and

$$
\|g\|_{C_{\gamma, \ln }[a, b]} \leq \max \left\{\|g\|_{C_{\gamma, \ln }[a, c]},\left(\ln \frac{b}{a}\right)^{\gamma}\|g\|_{C[c, b]}\right\} \text {. }
$$

Proof. The proof is similar to the proof of Lemma 3.1. Since $g \in C_{\gamma, \ln }[a, c]$ and $g \in C[c, b]$, we have $g \in C(a, b]$, that is, $g \in C_{\gamma, \ln }[a, b]$.

Next we give the estimate. Because $g \in C_{\gamma, \ln }[a, b]$, there exists at least $x^{*} \in[a, b]$ such that

$$
\|g\|_{C_{\gamma, \mathrm{ln}}[a, b]}=\left|\left(\ln \frac{x^{*}}{a}\right)^{\gamma} g\left(x^{*}\right)\right|
$$

If $x^{*} \in[a, c]$, then

$$
\|g\|_{C_{\gamma, \ln }[a, b]} \leq\|g\|_{C_{\gamma, \ln }[a, c]} .
$$

If $x^{*} \in[c, b]$, then

$$
\|g\|_{C_{r, \ln [a, b]}} \leq\left(\ln \frac{b}{a}\right)^{\gamma}\|g\|_{C[c, b]}
$$

Hence we have

$$
\|g\|_{C_{r, \mathrm{ln}}[a, b]} \leq \max \left\{\|g\|_{C_{\gamma, \ln }[a, c]},\left(\ln \frac{b}{a}\right)^{\gamma}\|g\|_{C[c, b]}\right\}
$$

This completes the proof of Lemma 3.4.

Next, on the basis of above lemmas, we establish the results about the existence and uniqueness of solution for the cauchy-type problem (2.10)-(2.11) in the sense of RiemannLiouville fractional derivative and Hadamard fractional derivative. 
Theorem 3.5. Let $\alpha>0$ and $n=-[-\alpha]$. Let $f:(a, b] \times \mathbb{R} \rightarrow \mathbb{R}$ be a function such that $f[x, y(x)] \in$ $C_{n-\alpha}[a, b]$ for any $y(x) \in C_{n-\alpha}[a, b]$ and the Lipschitzian condition holds with respect to the second variable $y$. Then there exists a unique solution $y(x) \in C_{n-\alpha}^{\alpha}[a, b]$ for the cauchy-type problem (2.10)(2.11).

Proof. First we prove the existence of a unique solution $y(x) \in C_{n-\alpha}[a, b]$. According to Theorem 2.8, it is sufficient to prove the existence of a unique solution $y(x) \in C_{n-\alpha}[a, b]$ to the nonlinear Volterra integral equation (2.12). Equation (2.12) makes sense in any interval $\left(a, x_{1}\right] \subset(a, b]\left(a<x_{1}<b\right)$. Choose $x_{1}$ such that

$$
A\left(x_{1}-a\right)^{\alpha} \frac{\Gamma(\alpha-n+1)}{\Gamma(2 \alpha-n+1)}<1
$$

where $A>0$ is the Lipschitzian coefficient. Next we prove the existence of a unique solution $y(x) \in C_{n-\alpha}\left[a, x_{1}\right]$ to $(2.12)$ on the interval $\left(a, x_{1}\right]$. For this, we use the Banach fixed point theorem for the space $C_{n-\alpha}\left[a, x_{1}\right]$, which is a complete metric space with the distance given by

$$
d\left(y_{1}, y_{2}\right)=\left\|y_{1}-y_{2}\right\|_{C_{n-\alpha}\left[a, x_{1}\right]}=\max _{x \in\left[a, x_{1}\right]}\left|(x-a)^{n-\alpha}\left[y_{1}(x)-y_{2}(x)\right]\right|
$$

We rewrite the integral (2.12) in the form

$$
y(x)=(T y)(x)
$$

where

$$
(T y)(x)=y_{0}(x)+\frac{1}{\Gamma(\alpha)} \int_{a}^{x} \frac{f[t, y(t)] d t}{(x-t)^{1-\alpha}}
$$

To apply Theorem 2.9, we have to prove the following: (1) if $y(x) \in C_{n-\alpha}\left[a, x_{1}\right]$, then $(T y)(x) \in C_{n-\alpha}\left[a, x_{1}\right] ;(2)$ for any $y_{1}, y_{2} \in C_{n-\alpha}\left[a, x_{1}\right]$ the following estimate holds:

$$
\left\|T y_{1}-T y_{2}\right\|_{C_{n-\alpha}\left[a, x_{1}\right]} \leq \omega\left\|y_{1}-y_{2}\right\|_{C_{n-\alpha}\left[a, x_{1}\right]}, \quad \omega=A\left(x_{1}-a\right)^{\alpha} \frac{\Gamma(\alpha-n+1)}{\Gamma(2 \alpha-n+1)}
$$

It follows from (2.13) that $y_{0}(x) \in C_{n-\alpha}\left[a, x_{1}\right]$. Since $f[x, y(x)] \in C_{n-\alpha}\left[a, x_{1}\right]$ for any $y(x) \in C_{n-\alpha}\left[a, x_{1}\right]$, then, by Lemma 3.2 [28] (with $\gamma=n-\alpha, b=x_{1}$, and $g(x)=f[x, y(x)]$ ), the integral in the right-hand side of (3.19) also belongs to $C_{n-\alpha}\left[a, x_{1}\right]$, and hence $(T y)(x) \in$ $C_{n-\alpha}\left[a, x_{1}\right]$. Now we prove the estimate in (3.21). By (3.20), using the Lipschitzian condition 
and applying the relation (3.6) (with $\gamma=n-\alpha, b=x_{1}$, and $g(x)=f\left[x, y_{1}(x)\right]-f\left[x, y_{2}(x)\right]$ ), we have

$$
\begin{aligned}
\left\|T y_{1}-T y_{2}\right\|_{C_{n-\alpha}\left[a, x_{1}\right]} & \leq\left\|I_{a+}^{\alpha}\left[\left|f\left[t, y_{1}(t)\right]-f\left[t, y_{2}(t)\right]\right|\right]\right\|_{C_{n-\alpha}\left[a, x_{1}\right]} \\
& \leq A\left\|I_{a+}^{\alpha}\left[\left|y_{1}(t)-y_{2}(t)\right|\right]\right\|_{C_{n-\alpha}\left[a, x_{1}\right]} \\
& \leq A\left(x_{1}-a\right)^{\alpha} \frac{\Gamma(\alpha-n+1)}{\Gamma(2 \alpha-n+1)}\left\|y_{1}-y_{2}\right\|_{C_{n-\alpha}\left[a, x_{1}\right]}
\end{aligned}
$$

which yields the estimate (3.21). In accordance with (3.17), $0<\omega<1$, and hence, by Theorem 2.9, there exists a unique solution $y^{*}(x) \in C_{n-\alpha}\left[a, x_{1}\right]$ to $(2.12)$ on the interval $\left[a, x_{1}\right]$.

By Theorem 2.9, this solution $y^{*}(x)$ is a limit of a convergent sequence $\left(T^{m} y_{0}^{*}\right)(x)$ :

$$
\lim _{m \rightarrow \infty}\left\|T^{m} y_{0}^{*}-y^{*}\right\|_{C_{n-\alpha}\left[a, x_{1}\right]}=0,
$$

where $y_{0}^{*}(x)$ is any function in $C_{n-\alpha}\left[a, x_{1}\right]$. If there is at least one $b_{k} \neq 0$ in the initial condition (2.11), then we can take $y_{0}^{*}(x)=y_{0}(x)$ with $y_{0}(x)$ defined by (2.13). The last relation can be rewritten into the form

$$
\lim _{m \rightarrow \infty}\left\|y_{m}-y^{*}\right\|_{C_{n-\alpha}\left[a, x_{1}\right]}=0,
$$

where

$$
y_{m}(x)=\left(T^{m} y_{0}^{*}\right)(x)=y_{0}(x)+\frac{1}{\Gamma(\alpha)} \int_{a}^{x} \frac{f\left[t,\left(T^{m-1} y_{0}^{*}\right)(t)\right] d t}{(x-t)^{1-\alpha}}, \quad(m \in N)
$$

Next we consider the interval $\left[x_{1}, b\right]$. Rewrite $(2.12)$ in the form

$$
y(x)=y_{01}(x)+\frac{1}{\Gamma(\alpha)} \int_{x_{1}}^{x} \frac{f[t, y(t)] d t}{(x-t)^{1-\alpha}}
$$

where $y_{01}(x)$ is defined by

$$
y_{01}(x)=\sum_{j=1}^{n} \frac{b_{j}}{\Gamma(\alpha-j+1)}(x-a)^{\alpha-j}+\frac{1}{\Gamma(\alpha)} \int_{a}^{x_{1}} \frac{f[t, y(t)] d t}{(x-t)^{1-\alpha}}
$$

We obtain $y_{01}(x) \in C\left[x_{1}, b\right]$. Next we prove the existence of a unique solution $y(x) \in C\left[x_{1}, b\right]$ to $(2.12)$ on the interval $\left[x_{1}, b\right]$. For this, we also use Banach fixed point theorem for the space $C\left[x_{1}, x_{2}\right]$, where $x_{2}$ satisfies

$$
\frac{A\left(x_{2}-x_{1}\right)^{\alpha}}{\alpha \Gamma(\alpha)}<1
$$


$C\left[x_{1}, x_{2}\right]$ is a complete metric space with the distance given by

$$
d\left(y_{1}-y_{2}\right)=\left\|y_{1}-y_{2}\right\|_{C\left[x_{1}, x_{2}\right]}=\max _{x \in\left[x_{1}, x_{2}\right]}\left|y_{1}(x)-y_{2}(x)\right|
$$

We rewrite the integral equation (3.26) into the form

$$
y(x)=(T y)(x)
$$

where

$$
(T y)(x)=y_{01}(x)+\frac{1}{\Gamma(\alpha)} \int_{x_{1}}^{x} \frac{f[t, y(t)] d t}{(x-t)^{1-\alpha}}
$$

To apply Theorem 2.9, we have to prove the following: (1) if $y(x) \in C\left[x_{1}, x_{2}\right]$, then $(T y)(x) \in C\left[x_{1}, x_{2}\right] ;(2)$ for any $y_{1}, y_{2} \in C\left[x_{1}, x_{2}\right]$, the following estimate holds:

$$
\left\|T y_{1}-T y_{2}\right\|_{C\left[x_{1}, x_{2}\right]} \leq \omega\left\|y_{1}-y_{2}\right\|_{C\left[x_{1}, x_{2}\right]^{\prime}} \quad \omega=\frac{A\left(x_{2}-x_{1}\right)^{\alpha}}{\alpha \Gamma(\alpha)}
$$

Since $f[x, y(x)] \in C_{n-\alpha}[a, b]$ for any $y(x) \in C_{n-\alpha}[a, b]$, then, by Lemma 3.3, the integral in the right-hand side of (3.31) also belongs to $C\left[x_{1}, x_{2}\right]$, and hence $(T y)(x) \in$ $\mathrm{C}\left[x_{1}, x_{2}\right]$. Now we prove the estimate in (3.32) as follows:

$$
\begin{aligned}
\left\|T y_{1}-T y_{2}\right\|_{C\left[x_{1}, x_{2}\right]} & \leq\left\|I_{a+}^{\alpha}\left[\left|f\left[t, y_{1}(t)\right]-f\left[t, y_{2}(t)\right]\right|\right]\right\|_{C\left[x_{1}, x_{2}\right]} \\
& \leq \frac{A\left(x_{2}-x_{1}\right)^{\alpha}}{\alpha \Gamma(\alpha)}\left\|y_{1}-y_{2}\right\|_{C\left[x_{1}, x_{2}\right]^{\prime}}
\end{aligned}
$$

which yields the estimate (3.32). In accordance with (3.28), then $0<\omega<1$, and hence by Theorem 2.9, there exists a unique solution $y_{1}^{*}(x) \in C\left[x_{1}, x_{2}\right]$ to $(2.12)$ on the interval $\left[x_{1}, x_{2}\right]$. By Theorem 2.9, this solution in $C\left[x_{1}, x_{2}\right]$ is a limit of a convergent sequence $\left(T^{m} y_{01}^{*}\right)(x)$ :

$$
\lim _{m \rightarrow \infty}\left\|T^{m} y_{01}^{*}-y_{1}^{*}\right\|_{C\left[x_{1}, x_{2}\right]}=0,
$$

where $y_{01}^{*}(x)$ is any function in $C\left[x_{1}, x_{2}\right]$. If $y_{0}(x) \neq 0$ on $\left[x_{1}, x_{2}\right]$, then we can take $y_{01}^{*}(x)=$ $y_{0}(x)$ with $y_{0}(x)$ defined by (2.13). The last relation can be rewritten in the form

$$
\lim _{m \rightarrow \infty}\left\|y_{m}-y_{1}^{*}\right\|_{C\left[x_{1}, x_{2}\right]}=0
$$

where

$$
y_{m}(x)=\left(T^{m} y_{01}^{*}\right)(x)=y_{01}(x)+\frac{1}{\Gamma(\alpha)} \int_{x_{1}}^{x} \frac{f\left[t,\left(T^{m-1} y_{01}^{*}\right)\right] d t}{(x-t)^{1-\alpha}}, \quad(m \in N)
$$


Next we consider the interval $\left[x_{2}, x_{3}\right]$, where $x_{3}=x_{2}+h_{2}$ such that $x_{3} \leq b$ and $\left(A\left(x_{3}-x_{2}\right)^{\alpha} / \alpha \Gamma(\alpha)\right)<1$. Using the same arguments as the above, we derive that there exists a unique solution $y_{2}^{*}(x) \in C\left[x_{2}, x_{3}\right]$ to $(2.12)$ on the interval $\left[x_{2}, x_{3}\right]$. If $x_{3} \neq b$, then take the next interval $\left[x_{3}, x_{4}\right]$, where $x_{4}=x_{3}+h_{3}$ and $h_{3}>0$ such that $x_{4} \leq b$ and $\left(A\left(x_{4}-x_{3}\right)^{\alpha} / \alpha \Gamma(\alpha)\right)<1$. If $x_{4}<b$, repeating the above process, then we find that there exists a unique solution $y(x)$ to (2.12), $y(x)=y_{k}^{*}(x)$, and $y_{k}^{*}(x) \in C\left[x_{k}, x_{k+1}\right](k=1, \ldots, L)$, where $x_{0}<x_{1}<\cdots<x_{L+1}=b$ and $\left(A\left(x_{k+1}-x_{k}\right)^{\alpha} / \alpha \Gamma(\alpha)\right)<1$, and we take $y_{0}(x)=y_{0 k}(x)$, and $y_{0}^{*}(x)=y_{0 k}^{*}(x)(k=1, \ldots, L)$ on each interval $\left[x_{k}, x_{k+1}\right]$. By $\left(A\left(x_{k+1}-x_{k}\right)^{\alpha} / \alpha \Gamma(\alpha)\right)<1$, we know that by finite steps we can arrive at $x_{L+1}=b$.

Then there exists a unique solution $y(x) \in C\left[x_{1}, b\right]$ to $(2.12)$ on the interval $\left[x_{1}, b\right]$. By Lemma 3.1, we obtain that there exists a unique solution $y(x) \in C_{n-\alpha}[a, b]$ to the Volterra integral equation (2.12) on the whole interval $[a, b]$, and hence $y(x) \in C_{n-\alpha}[a, b]$ is the unique solution to the cauchy-type problem (2.10)-(2.11).

To complete the proof of Theorem 3.5, we must show that such a unique solution $y(x) \in C_{n-\alpha}[a, b]$ belongs to the the space $C_{n-\alpha}^{\alpha}[a, b]$; it is sufficient to prove that $\left(D_{a+}^{\alpha} y\right)(x) \in$ $C_{n-\alpha}[a, b]$. By the above proof, the solution $y(x) \in C_{n-\alpha}[a, b]$ is a limit of the sequence $y_{m}(x)$, where $y_{m}(x)=\left(T^{m} y_{0}^{*}\right) \in C_{n-\alpha}[a, b]$ :

$$
\lim _{m \rightarrow \infty}\left\|y_{m}-y\right\|_{C_{n-\alpha}[a, b]}=0,
$$

with the choice of certain $y_{0}^{*}(x)$ on each $\left[a, x_{1}\right], \ldots,\left[x_{L}, b\right]$.

If $y_{0}(x) \neq 0$, then we can take $y_{0}^{*}(x)=y_{0}(x)$.

By (2.10) and the Lipschitzian-condition, we have

$$
\left\|D_{a+}^{\alpha} y_{m}-D_{a+}^{\alpha} y\right\|_{C_{n-\alpha}}=\left\|f\left[x, y_{m}\right]-f[x, y]\right\|_{C_{n-\alpha}} \leq A\left\|y_{m}-y\right\|_{C_{n-\alpha}} .
$$

Thus

$$
\lim _{m \rightarrow \infty}\left\|D_{a+}^{\alpha} y_{m}-D_{a+}^{\alpha} y\right\|_{C_{n-\alpha}}=0
$$

By $\left(D_{a+}^{\alpha} y_{m}\right)(x)=f\left[x, y_{m-1}(x)\right]$ and $f[x, y(x)] \in C_{n-\alpha}[a, b]$ for any $y(x) \in C_{n-\alpha}[a, b]$, we have $f\left[x, y_{m-1}(x)\right] \in C_{n-\alpha}[a, b]$, that is, $\left(D_{a+}^{\alpha} y_{m}\right)(x) \in C_{n-\alpha}[a, b]$. Hence $\left(D_{a+}^{\alpha} y\right)(x) \in$ $C_{n-\alpha}[a, b]$.

This completes the proof of Theorem 3.5.

Corollary 3.6. Let $\alpha>0$ and $n=-[-\alpha]$. Let $f:(a, b] \times \mathbb{R} \rightarrow \mathbb{R}$ be a function such that $f(x, y) \in C_{n-\alpha}[a, b]$ for any $y \in \mathbb{R}$; the Lipschitzian condition holds with respect to $y$ and $\lim _{x \rightarrow a}(x-a)^{n-\alpha} f(x, y(x))$, and $\lim _{x \rightarrow a}(x-a)^{n-\alpha} f(x, y(x))$ exist for any $y(x) \in C_{n-\alpha}[a, b]$. Then there exists a unique solution $y(x) \in C_{n-\alpha}^{\alpha}[a, b]$ for the cauchy-type problem (2.10)-(2.11).

Remark 3.7. It should be pointed out that the conditions in Theorem 3.5 are different from the ones in [28, Theorem 3.11, page 165]. In [28], $G$ is an open set in $R$ and $f$ is assumed to be a function such that $f[x, y] \in C_{n-\alpha}[a, b]$ for any $y \in G$. In fact, such assumptions are not complete for the proof of the related conclusion. A counterexample will be given in Section 4 . There exists the similar problem in [28, Theorem 3.29, page 213]. By applying Lemma 3.4, modifying the conditions in [28, Theorem 3.29, page 213] and using the similar arguments to the proof of Theorem 3.5, we arrive at the following result. 
Theorem 3.8. Let $\alpha>0, n=-[-\alpha]$, and $0 \leq \gamma<1$ such that $\gamma \geq n-\alpha$. Let $f:(a, b] \times \mathbb{R} \rightarrow \mathbb{R}(a>0)$ be a function such that $f[x, y(x)] \in C_{\gamma, \ln }[a, b]$ for any $y(x) \in C_{\gamma, \ln }[a, b]$ and the Lipschitzian condition holds with respect to $y$. Then there exists a unique solution $y(x)$ for the Cauchy-type problem

$$
\begin{aligned}
\left(\Phi_{a+}^{\alpha} y\right)(x) & =f[x, y(x)], \quad \alpha>0 ; x>a, \\
\left(\Phi_{a+}^{\alpha-k} y\right)(a+) & =b_{k}, \quad b_{k} \in \mathbb{R}(k=1, \ldots, n, n=-[-\alpha]) .
\end{aligned}
$$

in the space $C_{\delta ; n-\alpha, \gamma}^{\alpha}[a, b]$, where $\Phi_{a+}^{\alpha}$ is a Hadamard fractional derivative.

\section{Counterexamples}

In this section, by citing some counterexamples we would like to point out that, in [28, Lemmas 3.4, 3.9, and 3.10, pages 165, 202, and 213] are not complete.

Example 4.1. Let one consider the function

$$
g(x)= \begin{cases}\frac{\mu}{(x-a)^{\gamma}} & x \in(a, c], \\ \frac{\mu}{(x-c)^{\gamma}} & x \in(c, b],\end{cases}
$$

where $\mu \neq 0$ is a constant number and $\gamma \in(0, \infty)$.

From the above definition of $g(x)$, we know that $g(x) \in C_{\gamma}[a, c]$ and $g(x) \in C_{\gamma}[c, b]$, but we cannot get the conclusion $g(x) \in C_{\gamma}[a, b]$ and $\|g\|_{C_{Y}[a, b]} \leq \max \left\{\|g\|_{C_{Y}[a, c]},\|g\|_{C_{Y}[c, b]}\right\}$. Hence the conclusion of [28, Lemma 3.4, page 165] does not hold. We cannot apply it to prove [28, Theorem 3.11, page 165]. Furthermore, there also exists a problem about $f$ in [28, Theorem 3.11, page 165]. For example, choosing $f(x, y)=y \sin (1 /(x-a))$, we know that $f(x, y) \in C_{\gamma}[a, b]$ for any $y, f(x, y)$ satisfies Lipschitz condition with respect to the second variable $y$. However, choosing $y(x)=1 /(x-a)^{\gamma}$, we can not arrive at $(x-a)^{\gamma} f(x, y(x))=$ $\sin (1 /(x-a)) \in C[a, b]$. Hence the condition of $f$ in [28, Theorem 3.11, page 165] is not proper. 202].

The next example illustrates that there also exists a problem in [28, Lemma 3.9, page

Example 4.2. Consider the function

$$
\phi(x)=\ln x, \quad x \in[a, b], 1<a<b .
$$

It is evident that $\phi(x)$ belongs to the space $C^{1}[a, b]=\left\{\psi(x): \psi^{(1)}(x) \in C[a, b],\|\psi\|_{C^{1}[a, b]}\right.$ $\left.=\|\psi\|_{C[a, b]}+\left\|\psi^{(1)}\right\|_{C[a, b]}\right\}$.

Setting $c \in(a, b)$, that is, $a<c<b$, then

$$
\phi(x) \in C^{1}[a, b], \quad \phi(x) \in C^{1}[a, c], \quad \phi(x) \in C^{1}[c, b] .
$$


We could not conclude that

$$
\|\phi\|_{C^{1}[a, b]} \leq \max \left\{\|\phi\|_{C^{1}[a, c]},\|\phi\|_{C^{1}[c, b]}\right\}
$$

because

$$
\|\phi\|_{C^{1}[a, b]}=\ln b+\frac{1}{a}, \quad\|\phi\|_{C^{1}[a, c]}=\ln c+\frac{1}{a}, \quad\|\phi\|_{C^{1}[c, b]}=\ln b+\frac{1}{c} .
$$

However, we have

$$
\|\phi\|_{C^{1}[a, b]} \leq\|\phi\|_{C^{1}[a, c]}+\|\phi\|_{C^{1}[c, b]} .
$$

The following example is for [28, Lemma 3.10, page 213].

Example 4.3. Let one consider the function

$$
h(x)= \begin{cases}\frac{\mu}{(\ln x / a)^{\gamma}} & x \in(a, c], \\ \frac{\mu}{(\ln x / c)^{\gamma}} & x \in(c, b],\end{cases}
$$

where $\mu \neq 0$ is a constant number and $\gamma \in(0, \infty)$.

The same problem exists in [28, Lemma 3.10, page 213]. From the definition of $h(x)$, we have $h(x) \in C_{\gamma, \ln }[a, c]$ and $h(x) \in C_{\gamma, \ln }[c, b]$. However, the conclusion that $h(x) \in C_{\gamma, \ln }[a, b]$ and $\|h\|_{C_{Y}[a, b]} \leq \max \left\{\|h\|_{C_{Y}[a, c]},\|h\|_{C_{Y}[c, b]}\right\}$ is still not correct. This defect means that [28, Lemma 3.10] could not be applied to prove [28, Theorem 3.29].

In a sense, our lemmas and main results have remedied these defects.

\section{Conclusion}

In this paper, we first get several useful lemmas, especially Lemmas 3.1 and 3.4, which have improved the corresponding lemmas in [28]. By modifying the conditions on $f$ and improving the method used in [28], we have established the results of existence and uniqueness of solution for the cauchy-type problems involving the Riemann-Liouville fractional derivative and the Hadamard fractional derivative in the weight space of continuous functions. Meanwhile, we have given some counterexamples to prove that [28, Lemmas 3.4, 3.9, and 3.10, pages 165, 203, and 213] are not complete, which means that there exist some defects in the proofs of the related results in [28].

\section{Acknowledgments}

The authors would like to thank the anonymous referee for his/her remarks about the evaluation of the original version of the manuscript. This work is supported by the National Natural Science Foundation of China (Grant no. 10701023 and no. 10971221), the Natural 
Science Foundation of Shanghai (no. 10ZR1400100), and Chinese Universities Scientific Fund (B08-1).

\section{References}

[1] B. Bonilla, M. Rivero, L. Rodríguez-Germá, and J. J. Trujillo, "Fractional differential equations as alternative models to nonlinear differential equations," Applied Mathematics and Computation, vol. 187, no. 1, pp. 79-88, 2007.

[2] A. D. Fitt, A. R. H. Goodwin, K. A. Ronaldson, and W. A. Wakeham, "A fractional differential equation for a MEMS viscometer used in the oil industry," Journal of Computational and Applied Mathematics, vol. 229, no. 2, pp. 373-381, 2009.

[3] E. Ahmed and A. S. Elgazzar, "On fractional order differential equations model for nonlocal epidemics," Physica A: Statistical Mechanics and its Applications, vol. 379, no. 2, pp. 607-614, 2007.

[4] Y. Ding and H. Ye, "A fractional-order differential equation model of HIV infection of CD4+ T-cells," Mathematical and Computer Modelling, vol. 50, no. 3-4, pp. 386-392, 2009.

[5] H. Xu, "Analytical approximations for a population growth model with fractional order," Communications in Nonlinear Science and Numerical Simulation, vol. 14, no. 5, pp. 1978-1983, 2009.

[6] K. M. Furati and N.-E. Tatar, "On chaos synchronization of fractional differential equations," Journal of Mathematical Analysis and Applications, vol. 332, no. 1, pp. 441-454, 2007.

[7] J. Yan and C. Li, "Long time behavior for a nonlinear fractional model," Chaos, Solitons E Fractals, vol. 32, no. 2, pp. 725-735, 2007.

[8] C. Kou, Y. Yan, and J. Liu, "Stability analysis for fractional differential equations and their applications in the models of HIV-1 infection," Computer Modeling in Engineering \& Sciences, vol. 39, no. 3, pp. 301317, 2009.

[9] F. Mainardi, "Fractional calculus: some basic problems in continuum and statistical mechanics," in Fractals and Fractional Calculus in Continuum Mechanics (Udine, 1996), vol. 378 of CISM Courses and Lectures, pp. 291-348, Springer, Vienna, Austria, 1997.

[10] R. Metzler and T. F. Nonnenmacher, "Fractional diffusion: exact representations of spectral functions," Journal of Physics A, vol. 30, no. 4, pp. 1089-1093, 1997.

[11] R. Metzler and T. F. Nonnenmacher, "Fractional diffusion, waiting-time distributions, and Cattaneotype equations," Physical Review E, vol. 57, no. 6, pp. 6409-6414, 1998.

[12] W. M. Glöckle, R. Metzler, and T. F. Nonnenmacher, "Fractional model equation for anomalous diffusion," Physica A, vol. 211, pp. 13-24, 1994.

[13] B. J. West, P. Grigolini, R. Metzler, and T. F. Nonnenmacher, "Fractional diffusion and Levy stable processes," Physical Review E, vol. 55, no. 1, part A, pp. 99-106, 1997.

[14] H. E. Roman and M. Giona, "Fractional diffusion equation on fractals: three-dimensional case and scattering function," Journal of Physics A, vol. 25, no. 8, pp. 2107-2117, 1992.

[15] T. F. Nonnenmacher and D. J. F. Nonnenmacher, "Towards the formulation of a nonlinear fractional extended irreversible thermodynamics," Acta Physica Hungarica, vol. 66, no. 1-4, pp. 145-154, 1989.

[16] E. Pitcher and W. E. Sewell, "Existence theorems for solutions of differential equations of non-integral order," Bulletin of the American Mathematical Society, vol. 44, no. 2, pp. 100-107, 1938.

[17] J. H. Barrett, "Differential equations of non-integer order," Canadian Journal of Mathematics, vol. 6, no. 4, pp. 529-541, 1954.

[18] P. L. Butzer and A. A. Kilbas, "Mellin transform analysis and integration by parts for Hadamard-type fractional integrals," Journal of Mathematical Analysis and Applications, vol. 270, no. 1, pp. 1-15, 2002.

[19] P. L. Butzer, A. A. Kilbas, and J. J. Trujillo, "Fractional calculus in the Mellin setting and Hadamardtype fractional integrals," Journal of Mathematical Analysis and Applications, vol. 269, no. 1, pp. 1-27, 2002.

[20] P. L. Butzer, A. A. Kilbas, and J. J. Trujillo, “Compositions of Hadamard-type fractional integration operators and the semigroup property," Journal of Mathematical Analysis and Applications, vol. 269, no. 2, pp. 387-400, 2002.

[21] A. A. Kilbas, "Hadamard-type fractional calculus," Journal of the Korean Mathematical Society, vol. 38, no. 6, pp. 1191-1204, 2001.

[22] A. A. Kilbas, O. I. Marichev, and S. G. Samko, Fractional Integrals and Derivatives: Theory and Applications, Gordon and Breach Science Publishers, Amsterdam, The Netherlands, 1993.

[23] A. A. Kilbas and J. J. Trujillo, Hadamard-Type Fractional Integrals and Derivatives, vol. 11, Trudy Instituta Matematiki, Minsk, Russia, 2002. 
[24] H. A. H. Salem, "On the existence of continuous solutions for a singular system of non-linear fractional differential equations," Applied Mathematics and Computation, vol. 198, no. 1, pp. 445-452, 2008.

[25] A. Bashir and J. N. Juan, "Existence results for a coupled system of nonlinear fractional differential equations with three-point boundary conditions," Computers \& Mathematics with Applications, vol. 58, no. 9, pp. 1838-1843, 2009.

[26] G. Mehdi, "Solution of nonlinear fractional differential equations using homotopy analysis method," Applied Mathematical Modelling, vol. 58, no. 9, pp. 1838-1843, 2009.

[27] O. Abdulaziz, I. Hashim, and S. Momani, "Solving systems of fractional differential equations by homotopy-perturbation method," Physics Letters A, vol. 372, no. 4, pp. 451-459, 2008.

[28] A. A. Kilbas, H. M. Sprivastava, and J. J. Trujillo, Theory and Applications of Fractional Differential Equations, Elsevier, San Diego, Calif, USA, 2006. 


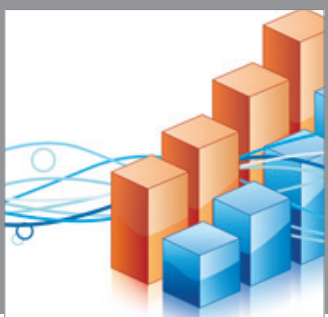

Advances in

Operations Research

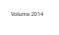

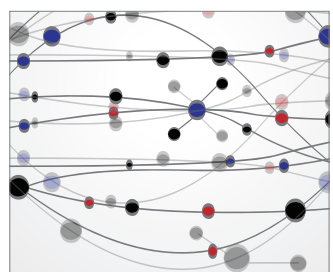

\section{The Scientific} World Journal
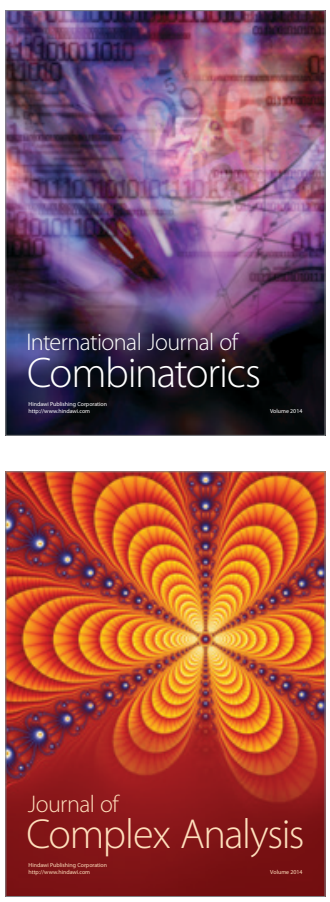

International Journal of

Mathematics and

Mathematical

Sciences
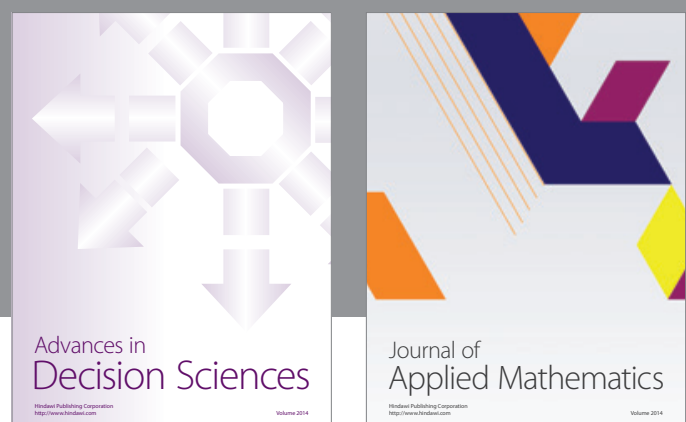

Journal of

Applied Mathematics
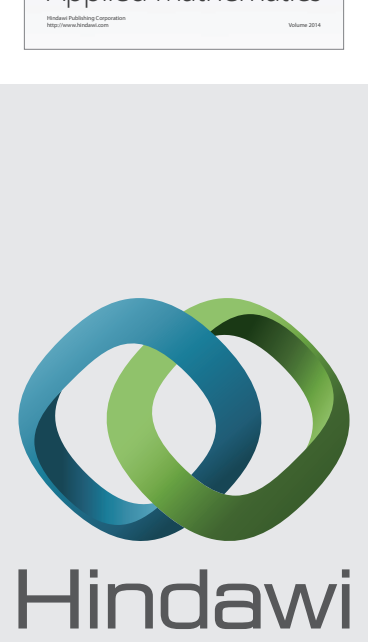

Submit your manuscripts at http://www.hindawi.com
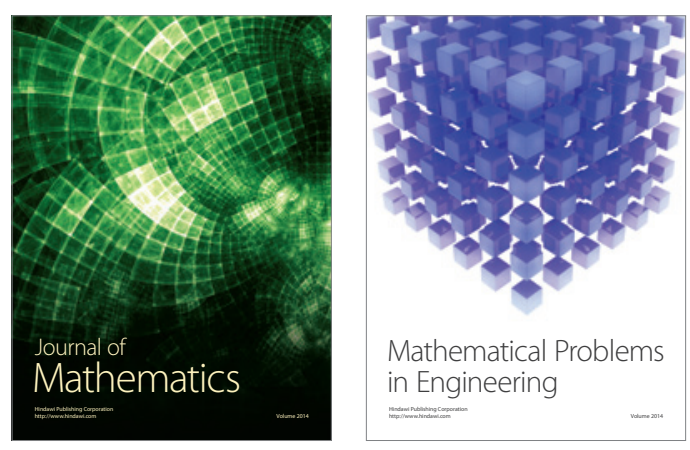

Mathematical Problems in Engineering
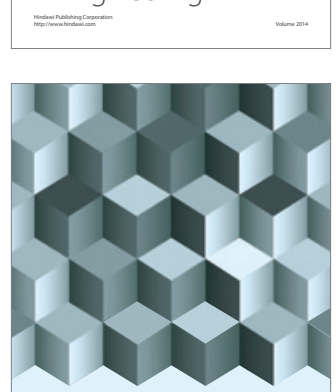

Journal of

Function Spaces
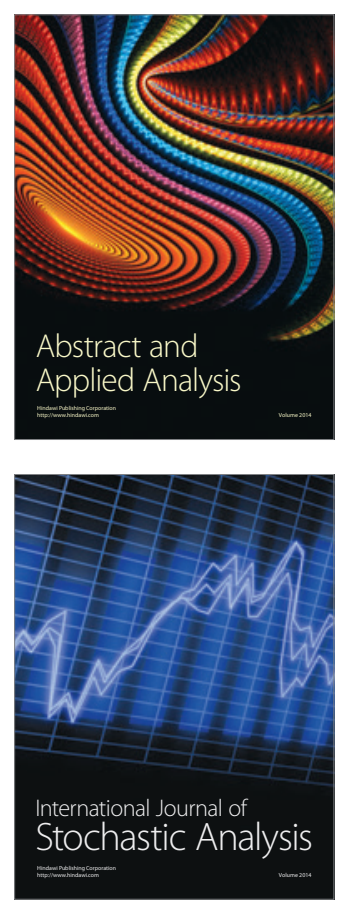

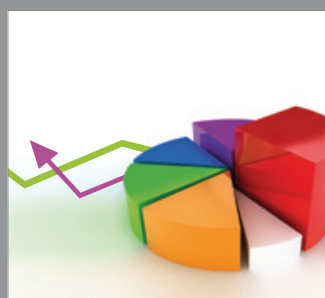

ournal of

Probability and Statistics

Promensencen
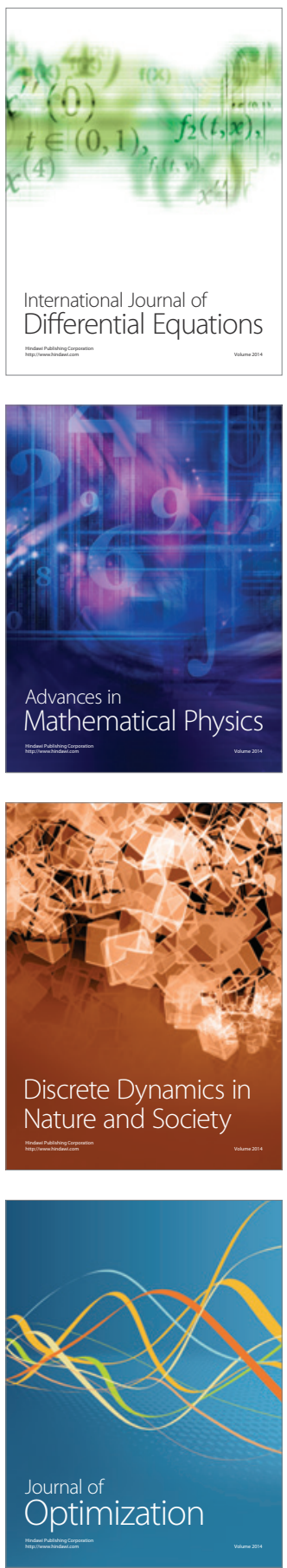